\section{NEONATAL SEPSIS}

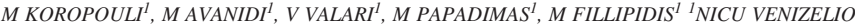
GENERAL HOSPITAL IRAKLIO (GREECE)

Neonatal sepsis continues to speculate neonatologists all over the world, while it remains the prime cause of mortality and morbility of neonates, and especialy the preterms.

Aim: Was to enrole sepsis, the pathogenic microbes and their sensitivities and especially their characteristics as the time of their appearence (precocious or belated) and the place (nosocomial or not).

Method: From 1/1/2003 to 28/2/2004 (14 months) we recorded the infections with positive blood cultures. Our material was 863 neonates who were hospitalised in our NICU.

Results: 654 out of blood cultures that were sent, 35 were positive in 32 neonates. The frequency of sepsis was $6,21 \%$. The mortality was $6 \%$ ( 2 neonates) $15 / 32$ neonates were $<35$ weeks. 12 neonate were infected by nosocomial infection and all of them were preterm. The pathogenetic organism wa gram (-) bacteria (8/12). The most common microbe was gram $(+)$ coccus (staphylococcus). 19 neonates were affected from $\mathrm{kl}$. Pneumoniae. All gram $(+)$ were sensitive to Vancomycin and all gram (-) to Imipenem, Amicasin and Ciprofloxacin. 15/32 neonates had the infection in the first $72 \mathrm{hrs}$ of life and all were gram $(+)$ except one. 12/15 were full term neonates. Premature rupture of membranes had only two neonates. There was not found any relationship between: sepsis and diabetes mellitus of the mother, multiple pregnancies, meconium aspiration, sex, and way of delivery.

Discusion and Conclusion: The resistant to oxacyclin staphylococcus aureus and epidermdis as well as the multiresistant gram (-) bacteria, are the most frequent bacteria of sepsis in our unit. The nosocomial infections are usually caused by klebsiella (ESBL). The early onset sepsis is caused almost exclusively by Gram $(+)$ microbes and have to do with full term neonates.

\section{8}

\section{BONE MORPHOGENETIC PROTEINS GENES AND NEURAL TUBE DE-}

\section{FECTS}

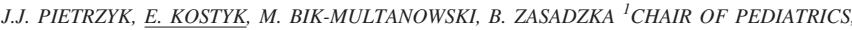
JAGIELLONIAN UNIVERSITY (POLAND)

Neural tube defects (NTD) are among the most common and severe congenital defects. The genetic background of a failure in the neural tube closure, which is the cause of NTD remains unknown. Among potential candidate genes which may contribute to the etiology of these defects the family of BMP (bone morphogenetic proteins) genes is considered. The aim of the study was to analyze the possible causative role of mutations of the BMP-2, BMP-4 and BMP-9 genes in the development of NTD.

Methods: Genomic sequences of the BMP-2, BMP-4 and BMP-9 genes were amplified by means of PCR technique and subsequently screened for mutations (denaturating high-performance liquid chromatography - DHPLC) in children with NTD. DNA fragments showing an abnormal DHPLC pattern were further directly sequenced to confirm the presence of the mutation. Subsequently, the frequency of the variant was analyzed and compared between the tested group of patients and the control group.

Results: 112 children with NTD were enrolled to the study. The control group consisted of 110 healthy children. No mutations were detected within the BMP-2 gene and 1 silent polymorphism (C>T transition in the 142 nd codon of the 2 nd exon of the gene, no change in protein sequence) was found in the BMP-9 gene. In the BMP-4 gene, the polymorphism was found $(538 \mathrm{~T}>\mathrm{C}$ transition with subsequent change of the protein sequence: 147 Valine $>$ Alanine). The frequency of $538 \mathrm{CC}$ homozygotes in the NTD group was higher (34.8\%) than in the controls (26.4\%), but the difference did no reach statistical significance (odds ratio $1.49 ; 95 \%$ confidence interval $0.84-2.63 ; \mathrm{p}$ value in chi-square test 0.17 ).

Conclusion: No mutations within the BMP-2, BMP-4 and BMP-9 genes were detected. The role of these genes in the development of NTD remains uncertain. The study was sponsored by a grant from State Committee for Scientific Research (No. 3P05E13823).

\section{9}

EXECUTIVE FUNCTIONS AND CEREBRAL MRI IN ADOLESCENTS WITH VERY LOW BIRTH WEIGHT

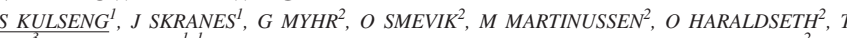
$V_{V I K^{3}}$, AM BRUBAKK ${ }^{l}$ DEP OF LAB. MEDICINE, CHILDRENS AND WOMENS HEALTH, ${ }^{2}$ DEP OF CIRCULATION AND IMAGING, ${ }^{3}$ DEP OF COMMUNITY MEDICINE AND GENERAL PRACTICE (NORWAY)

Background/Aim: High prevalence of abnormal cerebral MRI findings caused by perinatal periventricular leukomalacia (PVL) as well as disadvantages in executive functions has been reported in adolescents born prematurely. This study explores whether executive functions relates to cerebral MRI findings in these adolescents.

Methods: At age 14, 54 adolescents with birth weight equal to or below 1500g (VLBW) and 66 controls were examined. Executive functions were assessed by Wisconsin Card Sorting Test (WCST). On cerebral MRI, dilation of ventricles, white matter reduction and corpus callosum thinning were reported and compared with results on WCST.

Results: $82 \%$ VLBW adolescents and 12\% controls had dilation of the ventricular system. On WCST, VLBW adolescents with dilated ventricles had more trials to complete 1 st category $(\mathrm{p}<0.05)$, made more errors of perseveration $(p<0.05)$ and non-perseveration $(p<0.01)$, and had a tendency to lower percentiles on learning to learn compared with those without ventricular dilation. Thinning of corpus callosum was related to more errors of perseveration $(\mathrm{p}<0.05)$ and non-perseveration $(\mathrm{p}<$ 0.01 ) and lower percentile score on learning to learn in the VLBW group $(p<0.05)$. VLBW adolescents with white matter reduction also made more errors of non-perseveration $(\mathrm{p}<0.05)$ and tended to have more errors of perseveration than VLBW adolescents without white matter reduction. These differences were not found within the controls.

Conclusion: Ventricular dilation, thinning of the corpus callosum and cerebral white matte reduction in VLBW adolescents were related to disadvantages in performances on problem solvin aspects of executive functions suggesting a less flexible and efficient problem solving strategy. We speculate whether this is due to perinatal PVL with subsequent loss of white matter tracts connectin occipital, parietal and temporal lobe functions to prefrontal areas. The long term consequences of los of projection, association and commissural fibres on higher order cognitive functions warrant further research.
CONGENITAL CYTOMEGALOVIRUS (CMV) INFECTION AND AGENESIS OF CORPUS CALLOSUM

P KUTZl', B SCHWEIGER ${ }^{2}$, C ROLL ${ }^{1}$ I DEPARTEMENT OF NEONATOLOGY OF UNIVERSITY, 'DEPARTEMENT OF RADIOLOGY OF UNIVERSITY (GERMANY)

Background: Congenital CMV infection is associated with a wide spectrum of neuropathology, ranging from inflammation to destruction and malformation. Disturbances of neuronal migration with polymicrogyria involving cerebellar and cerebral cortex, lissencephaly, pachygyria, and schizencephaly have been observed. We present a newborn with congenital CMV infection exhibiting agenesis of the corpus callosum.

Case presentation: Prenatal ultrasound at 32 wks gestational age showed corpus callosum agenesis in the fetus. Subsequent serologic tests detected primary CMV infection in the pregnant woman. The $2530 \mathrm{~g}$-girl was delivered at $39 \mathrm{wks}$. Besides a dysmorphic face with hypertelorism, no other anomalies were noted.

Results: Serology confirmed CMV infection, but CMV early antigen was negative and PCR for DNA positive in only one of several urine specimens indicating that infection was no longer active. Utrasound of the brain showed agenesis of the corpus callosum and hypoplasia of the vermis. MR additionally detected pachygyria. There was no CMV-retinitis and auditory brain stem responses were normal. Clinically, the girl showed feeding difficulties.

Conclusion: This is only the second report of corpus callosum agenesis in a newborn infant with congenital CMV infection. As the corpus callosum develops at approximately 10 weeks of gestation, the primary infection probably occurred in the first trimester, disturbing differentiation and migration of neuronal precursor cells.

\section{1}

VASCULAR ENDOTHELIAL GROWTH FACTOR AND INSULIN GROWTH FACTOR 1 SERUM CONCENTRATIONS IN VERY LOW BIRTHWEIGHT INFANTS WITH RETINOPATHY

P KWINTA ${ }^{l}$, Z MITKOWSKA ${ }^{l}, T$ TOMASIK ${ }^{l}$, JJ PIETRZYK ${ }^{l}$ IDEPARTMENT OF PEDIATRICS, IAGIELLONIAN UNIVERSITY (POLAND)

Background: Recent experimental studies suggest that growth factors such as vascular endothelia growth factor (VEGF) and insulin growth factor 1 (IGF-1) can play an important role in the development of retinopathy of prematurity (ROP), but clinical implications of these findings remain unclear.

Aim: Analysis of correlation betw month of life and the risk of ROP.

Methods: A sample of 56 newborns with mean birthweight 1095g (range: 600-1500g) were evaluated prospectively. Concentrations of VEGF and IGF-1 were measured by Elisa assay in the 2nd, 3rd and 4th week of life. The infants were divided into 3 groups: A) no ROP $(n=37)$, B) ROP no requiring treatment $(n=5)$ and 3$)$ ROP requiring laser or cryotherapy $(n=14)$.

Results: Significant, however low grade, correlations between postconceptional age and VEGF $(\mathrm{r}=0.2 ; \mathrm{p}=0.01)$ and IGF-1 $(\mathrm{r}=0.32 ; \mathrm{p}<0.001)$ concentrations were found. VEGF and IGF-1 concentrations in the group A infants were used to produce $z$-score tables of growth factors concentration according to postconceptional age. Compared to the group A newborns, mean $\mathrm{z}$-score of IGF-1 concentrations were significantly lower in the group B $(-0.29)$ and $\mathrm{C}$ infants $(-0.53 ; \mathrm{p}=0.02)$, but the mean z-score of VEGF concentrations were insignificantly higher ( 0.17 and 0.33 respectively; $\mathrm{p}=0.3$ )

Conclusion: VEGF and IGF-1 concentrations highly correlate with maturity of the infant. Low GF-1 concentrations during the first month of life can be associated with the risk of ROP.

Study partially supported by Polish Ministry of Science grant No 0642/P05/2003/24
RESULTS OF CONTINUOS MONITORING OF HEMOGLOBIN SATURATION DURING THE FIRST MONTH OF LIFE AS PREDICTORS OF RETINOPATHY OF PREMATURITY

$P$ KWINTA ${ }^{1}, P$ KRUCZEK ${ }^{1}, P$ STOCH ${ }^{2}, W$ WAJS $^{2}, J J$ PIETRZYK ${ }^{1}{ }^{1}$ DEPARTMENT OF PEDIAT RICS, JAGIELLONIAN UNIVERSITY ${ }^{2}$ INSTITUTE OF METALURGY AND MINING (POLAND)

Background: The results of experimental studies indicate that the relation between oxygen therapy and the stage of retinopathy of prematurity (ROP) cannot be described by a simple model. Technical progress, new methods of vital signs recording and transmission allow for better, continuous monitoring of the patients status.

Aim: Analysis of correlation between results of continuous monitoring of hemoglobin saturation during the first month of life and the risk of proliferative ROP. Methods: A sample of 26 newborn with mean birthweight $1060 \mathrm{~g}$ (range: $600-1450 \mathrm{~g}$ ) were evaluated prospectively. Oxygen hemoglobin saturation ( $\mathrm{spO} 2$ ) was monitored on the continuous basis and recorded in computer database during the first 4 weeks of life. The infants were divided into 2 groups: A) no ROP $(n=20)$, B) ROP requiring laser or cryotherapy $(n=6)$

Results: The recorded values during the first week of life were similar in both groups. The differences were recorded in the 2nd-4th week of life. Surprisingly, mean spO2 were higher in the group A (group A vs B: week 2: 96.5 vs $93.7 \%$, week 3: 95.9 vs $93.3 \%$, week: 4: 95.9 vs $93.5 \%$, $\mathrm{p}<0.02$ ) and the total time (percantage) with $\mathrm{spO} 2>97 \%$ ( 48 vs $17 \%, 40$ vs $20 \%, 41$ vs $21 \%$ ) was insignificantly higher. The total time (percentage) with $\mathrm{spO} 2<85 \%(1.2$ vs $3.3 \%, 1.7$ vs $6.6 \%, 1.7$ vs $7.7 \%$; $<<0,001)$, mean drop of $\mathrm{spO} 2$ from $85 \%$ (6.1 vs $6.7 \%, 4.6$ vs $6.8 \%, 4.8$ vs $6.8 \% ; \mathrm{p}=0.02)$ and standard deviation of recorded $\mathrm{spO} 2$ values reflecting $\mathrm{spO} 2$ fluctation ( 3 vs $4.3 \%, 3.4$ vs $5.6 \%, 3.3$ vs $5.9 \% ; \mathrm{p}=0.03$ ) were higher in the group B infants.

Conclusion: Based on our preliminary study, we recommend that paying special special attention to prevent hyperoxia, we should not forget about preventing hypoxia which seems to be also a major isk factor of ROP.

Study partially supported by Polish Ministry of Science grant No 0642/P05/2003/24 